\title{
RESTRAINED DOUBLE MONOPHONIC NUMBER OF A GRAPH ${ }^{1}$
}

\author{
A.P. Santhakumaran ${ }^{2}$ \\ Department of Mathematics, Hindustan Institute of Technology and Science, \\ Chennai - 603 103, India \\ apskumar1953@gmail.com \\ K. Ganesamoorthy \\ Department of Mathematics, Coimbatore Institute of Technology, \\ Coimbatore - 641 014, India \\ kvgm_2005@yahoo.co.in
}

\begin{abstract}
For a connected graph $G$ of order at least two, a double monophonic set $S$ of a graph $G$ is a restrained double monophonic set if either $S=V$ or the subgraph induced by $V-S$ has no isolated vertices. The minimum cardinality of a restrained double monophonic set of $G$ is the restrained double monophonic number of $G$ and is denoted by $d m_{r}(G)$. The restrained double monophonic number of certain classes graphs are determined. It is shown that for any integers $a, b, c$ with $3 \leq a \leq b \leq c$, there is a connected graph $G$ with $m(G)=a, m_{r}(G)=b$ and $d m_{r}(G)=c$, where $m(G)$ is the monophonic number and $m_{r}(G)$ is the restrained monophonic number of a graph $G$.
\end{abstract}

Keywords: Monophonic set, Restrained monophonic set, Restrained monophonic number, Restrained double monophonic set, Restrained double monophonic number.

AMS Subject Classification: $05 \mathrm{C} 12$.

\section{Introduction}

By a graph $G=(V, E)$ we mean a finite undirected connected graph without loops or multiple edges. The order and size of $G$ are denoted by $p$ and $q$ respectively. For basic graph theoretic terminology we refer to Harary [5]. For vertices $u$ and $v$ in a connected graph $G$, the distance $d(u, v)$ is the length of a shortest $u-v$ path in $G$. An $u-v$ path of length $d(u, v)$ is called an $u-v$ geodesic. It is known that $d$ is a metric on the vertex set $V$ of $G$ [2]. The neighborhood of a vertex $v$ is the set $N(v)$ consisting of all vertices $u$ which are adjacent with $v$. A vertex $v$ is an extreme vertex if the subgraph induced by its neighbors is complete.

The closed interval $I[x, y]$ consists of all vertices lying on some $x-y$ geodesic of $G$, while for $S \subseteq V, I[S]=\bigcup_{x, y \in S} I[x, y]$. A set $S$ of vertices of $G$ is a geodetic set if $I[S]=V$, and the minimum cardinality of a geodetic set is the geodetic number $g(G)$. The geodetic number of a graph was introduced in $[2,6]$ and further studied in [3, 4]. It was shown in [6] that determining the geodetic number of a graph is an NP-hard problem. A geodetic set $S$ of a graph $G$ is a restrained geodetic set if the subgraph $G[V-S]$ induced by $V-S$ has no isolated vertex. The minimum cardinality of a restrained geodetic set of $G$ is the restrained geodetic number of $G$. The restrained geodetic

\footnotetext{
${ }^{1}$ The second author research work was supported by National Board for Higher Mathematics, INDIA (Project No. NBHM/R.P.29/2015/Fresh/157).

${ }^{2}$ Former Professor
} 
number of a graph was introduced and studied in [1]. Let $2^{V}$ denote the set of all subsets of $V$. The mapping $I: V \times V \rightarrow 2^{V}$ defined by $I[u, v]=\{w \in V: w$ lies on a $u-v$ geodesic in $G\}$ is the interval function of $G$. One of the basic properties of $I$ is that $u, v \in I[u, v]$ for any pair $u, v \in V$. Hence the interval function captures every pair of vertices and so the problem of double geodetic sets is trivially well-defined while it is clear that this fails in many graphs already for triplets (for example, complete graphs). This motivated us to introduce and study double geodetic sets in [7] and further double monophonic sets in [9]. Also, double monophonic parameters like the upper double monophonic number of a graph and the connected double monophonic number of a graph were studied in $[10,11]$. This is the basis behind the introduction and study of the restrained double monophonic number of a graph. A set $S$ of vertices is called a double geodetic set of $G$ if for each pair of vertices $x, y$ in $G$ there exist vertices $u, v \in S$ such that $x, y \in I[u, v]$. The double geodetic number $d g(G)$ is the minimum cardinality of a double geodetic set. The double geodetic number of a graph was introduced and studied in [7].

A chord of a path $P$ is an edge joining two non-adjacent vertices of $P$. A path $P$ is called a monophonic path if it is a chordless path. A set $S$ of vertices of $G$ is a monophonic set of $G$ if each vertex $v$ of $G$ lies on an $x-y$ monophonic path for some $x, y \in S$. The minimum cardinality of a monophonic set of $G$ is the monophonic number of $G$ and is denoted by $m(G)$. The monophonic number of a graph was studied and discussed in [8]. A set $S$ of vertices of $G$ is called a double monophonic set of $G$ if for each pair of vertices $x, y$ in $G$ there exist vertices $u, v$ in $S$ such that $x, y$ lie on a $u-v$ monophonic path. The double monophonic number $d m(G)$ of $G$ is the minimum cardinality of a double monophonic set of $G$. The concept of double monophonic number of a graph was introduced and studied in [9].

The concept of distance in graphs is a major component in graph theory with its centrality and convexity concepts having numerous applications to real life problems. There are several interesting applications of these concepts to facility location in real life situations, routing of transport problems and communication network designs. As the paths involved in the discussion of this paper are monophonic, no intervention by hackers or enemies is possible to the respective facilities provided. Further, as monophonic paths are secured and longer than geodesic paths, it is advantageous to more customers in getting the service with protection.

The following theorems will be used in the sequel.

Theorem 1 [8]. Each extreme vertex of a connected graph $G$ belongs to every monophonic set of $G$.

Theorem 2 [8]. For the complete graph $K_{p}(p \geq 2), m\left(K_{p}\right)=p$.

Theorem 3 [7]. Each extreme vertex of a connected graph $G$ belongs to every double geodetic set of $G$.

Throughout this paper $G$ denotes a connected graph with at least two vertices.

\section{Restrained double monophonic number}

To study the main concepts of this paper, we introduce first the restrained monophonic number of a graph and the restrained double geodetic number of a graph, and then prove some basic results and proceed.

Definition 1. A restrained monophonic set $S$ of a graph $G$ is a monophonic set such that either $S=V$ or the subgraph induced by $V-S$ has no isolated vertices. The minimum cardinality of a restrained monophonic set of $G$ is the restrained monophonic number of $G$ and is denoted by $m_{r}(G)$. 
Example 1. For the cycle $C_{5}: u, v, w, x, y, u$ of order 5 , it is easily verified that $S=\{u, w\}$ is a minimum monophonic set of $C_{5}$ and so $m\left(C_{5}\right)=2$. Since the subgraph induced by $V-S$ has an isolated vertex $v, S$ is not a restrained monophonic set of $C_{5}$. It is clear that, $S \cup\{v\}$ is a minimum restrained monophonic set of $C_{5}$ so that $m_{r}\left(C_{5}\right)=3$. Thus the monophonic number and the restrained monophonic number of a graph are different.

It is clear that every restrained monophonic set of $G$ is a monophonic set of $G$ and so Theorem 1 gives the next result.

Theorem 4. Each extreme vertex of a connected graph $G$ belongs to every restrained monophonic set of $G$.

Corollary 1. For the complete graph $K_{p}(p \geq 2), m_{r}\left(K_{p}\right)=p$.

Definition 2. A double geodetic set $S$ of a graph $G$ is a restrained double geodetic set if either $S=V$ or the subgraph induced by $V-S$ has no isolated vertices. The minimum cardinality of a restrained double geodetic set of $G$ is the restrained double geodetic number of $G$ and is denoted by $d g_{r}(G)$.

Example 2. For the cycle $C_{4}$ of order 4, it is clear that any set $S$ of two non-adjacent vertices of $C_{4}$ is a minimum double geodetic set of $C_{4}$ and so $d g\left(C_{4}\right)=2$. Since the subgrph induced by $V-S$ has an isolated vertices, $S$ is not a restrained double geodetic set of $C_{4}$. Also, no 3-element subset of $V\left(C_{4}\right)$ is a restrained double geodetic set of $C_{4}$. Thus $V\left(C_{4}\right)$ is the unique minimum restrained double geodetic set of $C_{4}$ and so $d g_{r}\left(C_{4}\right)=4$. Hence the double geodetic number and the restrained double geodetic number of a graph are different.

It is clear that every restrained double geodetic set of $G$ is a double geodetic set of $G$ and so Theorem 3 gives the next result.

Theorem 5. Each extreme vertex of a connected graph $G$ belongs to every restrained double geodetic set of $G$.

Now, we introduce the restrained double monophonic number of a graph and investigate.

Definition 3. A double monophonic set $S$ of a graph $G$ is a restrained double monophonic set if either $S=V$ or the subgraph induced by $V-S$ has no isolated vertices. The minimum cardinality of a restrained double monophonic set of $G$ is the restrained double monophonic number of $G$ and is denoted by $d m_{r}(G)$.

Example 3. For the graph $G$ given in Fig. 1, it is clear that no 2-element subset of vertices of $G$ is a monophonic set of $G$. Since $S=\{v, x, z\}$ is a monophonic set of $G, m(G)=3$. It is the only minimum monophonic set of $G$. Since the subgraph induced by $V-S$ has the isolated vertex $v_{4}$, it is not a restrained monophonic set of $G$. Now, the set $S_{1}=\left\{v, x, z, v_{4}\right\}$ is a restrained monophonic set of $G$ so that $m_{r}(G)=4$.

It is easily seen that no 4-element subset of vertices of $G$ containing the vertex $v$ is a double monophonic set of $G$. Also, it is clear that the set $S_{2}=\left\{v, v_{1}, v_{2}, v_{3}, v_{4}\right\}$ is the unique minimum double monophonic set of $G$. Since the subgraph induced by $V-S_{2}$ has no isolated vertices, $S_{2}$ is a minimum restrained double monophonic set of $G$ so that $d m_{r}(G)=5$. Thus the monophonic number, the restrained monophonic number and the restrained double monophonic number of a graph are different. 


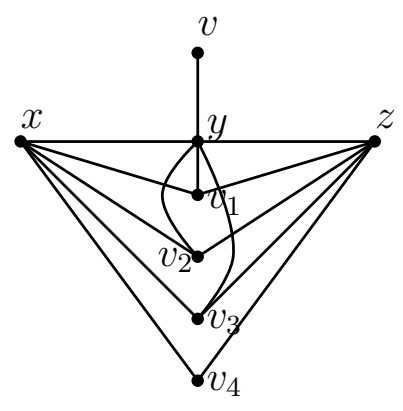

Figure 1. Graph $G$.

Theorem 6. Every extreme vertex of a connected graph $G$ belongs to every restrained double monophonic set of $G$. In particular, if the set of all extreme vertices of $G$ is a restrained double monophonic set, then it is the unique minimum restrained double monophonic set of $G$.

P r o o f. Since every restrained double monophonic set is a monophonic set, the result follows from Theorem 1.

The following results are easy consequences of Theorem 6 .

Result 1. For the complete graph $K_{p}(p \geq 2), d m_{r}(G)=p$.

Result 2. For a graph $G$ of order $p$ with $k$ extreme vertices, $\max \{2, k\} \leq d m_{r}(G) \leq p$.

Result 3. If $T$ is a tree of order $p$ with $k$ end-vertices and $p-k \geq 2$, then $d m_{r}(T)=k$.

Theorem 7. For any graph $G$ of order $p$,

$$
2 \leq m(G) \leq m_{r}(G) \leq d m_{r}(G) \leq p, \quad m_{r}(G) \neq p-1 \neq d m_{r}(G) .
$$

$\mathrm{P}$ r o o f. Any monophonic set needs at least two vertices and hence $m(G) \geq 2$. Since every restrained monophonic set is also a monophonic set of $G$, it follows that $m(G) \leq m_{r}(G)$. It is clear that every restrained double monophonic set of $G$ is also a restrained monophonic set and so $m_{r}(G) \leq d m_{r}(G)$. Since the set of all vertices of $G$ is a restrained double monophonic set of $G$, $d m_{r}(G) \leq p$. From the definitions of restrained monophonic number and the restrained double monophonic number, it is clear that $m_{r}(G) \neq p-1 \neq d m_{r}(G)$.

Remark 1. The bounds in Theorem 7 are sharp. The two end-vertices of a nontrivial path $P_{n}$ on $n$ vertices is its unique minimum monophonic set so that $m\left(P_{n}\right)=2$ and for the complete graph $K_{p}(p \geq 2)$, we have $d m_{r}\left(K_{p}\right)=p$. Also, all the inequalities in Theorem 7 can be strict, for the graph $G$ of order 8 given in Fig. $1, m(G)=3, m_{r}(G)=4$ and $d m_{r}(G)=5$. Thus we have $2<m(G)<m_{r}(G)<d m_{r}(G)<p$.

Theorem 8. For any graph $G$ of order $p, 2 \leq d m_{r}(G) \leq d g_{r}(G) \leq p, d m_{r}(G) \neq p-1 \neq$ $d g_{r}(G)$.

$\mathrm{P}$ r o o f. Any restrained double monophonic set needs at least two vertices and so $d m_{r}(G) \geq 2$. It is clear that every restrained double geodetic set of $G$ is also a restrained double monophonic set and so $d m_{r}(G) \leq d g_{r}(G)$. Since the set of all vertices of $G$ is a restrained double geodetic set of $G$, $d g_{r}(G) \leq p$. From the definitions of restrained double monophonic number and the restrained 
double geodetic number, it is clear that $d m_{r}(G) \neq p-1 \neq d g_{r}(G)$.

Remark 2. The bounds in Theorem 8 are sharp. For the path $P_{n}(n \geq 4), d m_{r}(G)=d g_{r}(G)=2$ and for the complete graph $K_{p}(p \geq 3), d m_{r}\left(K_{p}\right)=d g_{r}\left(K_{p}\right)=p$. All the inequalities in Theorem 8 can be strict. For the graph $G$ of order 7 given in Fig. 2, no 2-element subset of $V(G)$ forms a minimum restrained double monophonic set of $G$. The minimum restrained double monophonic sets of $G$ are $S_{1}=\left\{v_{1}, v_{2}, v_{5}\right\}$ and $S_{2}=\left\{v_{1}, v_{2}, v_{6}\right\}$ so that $d m_{r}(G)=3$. Also, there is no 3-element or 4-element subset of $V(G)$ forms a minimum restrained double geodetic set of $G$. It is easy to verify that $S_{3}=\left\{v_{1}, v_{2}, v_{4}, v_{5}, v_{6}\right\}$ is a minimum restrained double geodetic set of $G$ and so $d g_{r}(G)=5$. Thus we have $2<d m_{r}(G)<d g_{r}(G)<p$.

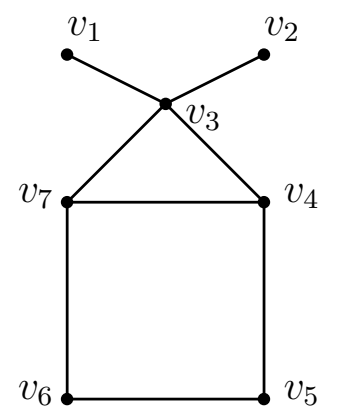

Figure 2. Graph $G$.

The following results are easy to prove.

Result 4. For any cycle $C_{p}, d m_{r}\left(C_{p}\right)= \begin{cases}p, & \text { if } p=3,4 \\ 3, & \text { if } p=5 \\ 2, & \text { if } p \geq 6\end{cases}$

Result 5. For any wheel $W_{p}=K_{1}+C_{p-1},(p \geq 4), d m_{r}\left(W_{p}\right)= \begin{cases}4, & \text { if } p=4, \\ 2, & \text { if } p \geq 5\end{cases}$

Result 6. For the complete bipartite graph $G=K_{m, n}(2 \leq m \leq n)$,

$$
d m_{r}(G)= \begin{cases}n+2, & \text { if } 2=m \leq n \\ 4, & \text { if } 3 \leq m \leq n\end{cases}
$$

In view of Theorem 7 , we have the following realization theorem.

Theorem 9. For any three integers $a, b, c$ with $3 \leq a \leq b \leq c$, there is a connected graph $G$ with $m(G)=a, m_{r}(G)=b$ and $d m_{r}(G)=c$.

P r o o f. This theorem is proved by considering four cases.

Case 1. $a=b=c$. Then, for the complete graph $G=K_{a}$, by Theorem 2, Corollary 1 and Result 1, $m(G)=m_{r}(G)=d m_{r}(G)=a$.

Case 2. $a=b<c$. Let $G$ be the graph in Fig. 3 is got by adding $a-1$ new vertices $w_{1}, w_{2}, \cdots, w_{a-2}, x$ to the complete bipartite graph $K_{2, c-a+1}$ with the partite sets $U=\left\{u_{1}, u_{2}\right\}$ and $W=\left\{v_{1}, v_{2}, \cdots, v_{c-a+1}\right\}$, joining each vertex $w_{i}(1 \leq i \leq a-2)$ to the vertex $u_{1}$ and joining the vertex $x$ to the vertex $v_{1}$. By Theorems 1, 4 and 6, every monophonic set, every restrained monophonic 
set and every restrained double monophonic set of $G$ contain the set $S=\left\{w_{1}, w_{2}, \cdots, w_{a-2}, x\right\}$ of all extreme vertices of $G$. Clearly, $S$ is not a monophonic set of $G$. It is easy to verify that $S_{1}=S \cup\left\{u_{2}\right\}$ is the unique minimum monophonic set of $G$ and so $m(G)=a$. Since the subgraph induced by $V-S_{1}$ has no isolated vertices, $S_{1}$ is the unique minimum restrained monophonic set of $G$ so that $m_{r}(G)=a=m(G)$. It is clear that the pair of vertices $x, v_{i}(i=2,3, \cdots, c-a+1)$ do not lie on any $u-v$ monophonic path, for any $u, v \in S_{1}$ and so $S_{1}$ is not a restrained double monophonic set of $G$. It is easy to verify that $S_{2}=S \cup\left\{u_{2}, v_{2}, \cdots, v_{c-a+1}\right\}$ is a minimum double monophonic set of $G$ and the subgraph induced by $V-S_{2}$ has no isolated vertices so that $d m_{r}(G)=c$.

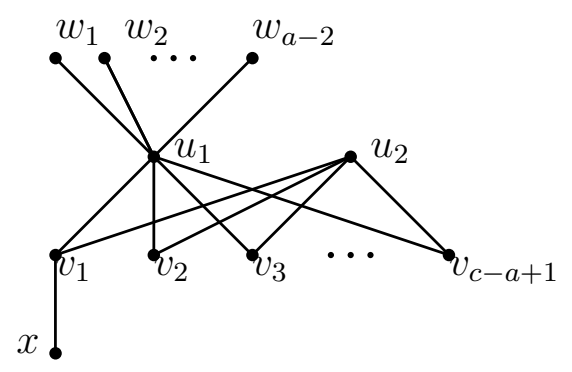

Figure 3. Graph $G$.

Case 3. $a<b=c$. Let $G$ be the graph in Fig. 4 formed from the path $P_{3}: v_{1}, v_{2}, v_{3}$ of order 3 , by adding $b$ new vertices $u_{1}, u_{2}, \cdots, u_{a-2}, w_{1}, w_{2}, \ldots, w_{b-a+2}$ to $P_{3}$ and joining each vertex $u_{i}(1 \leq i \leq a-2)$ to $v_{2}$; and joining each vertex $w_{j}(1 \leq j \leq b-a+2)$ to $v_{1}$ and $v_{3}$. By Theorems 1, 4 and 6, every monophonic set, every restrained monophonic set and every restrained double monophonic set of $G$ contain the set $S=\left\{u_{1}, u_{2}, \cdots, u_{a-2}\right\}$ of all extreme vertices of $G$. Clearly, $S$ is not a monophonic set of $G$. Also, for any $x \in V-S, S \cup\{x\}$ is not a monophonic set of $G$. It is easy to verify that $S_{1}=S \cup\left\{v_{1}, v_{3}\right\}$ is a minimum monophonic set of $G$ and so $m(G)=a$. Since the subgraph induced by $V-S_{1}$ has the isolated vertices $w_{1}, w_{2}, \cdots, w_{b-a+2}, v_{2}$, $S_{1}$ is not a restrained monophonic set of $G$. It is clear that, every restrained monophonic set and every restrained double monophonic set of $G$ contains $\left\{w_{1}, w_{2}, \cdots, w_{b-a+2}\right\}$ and it follows that $S_{2}=S \cup\left\{w_{1}, w_{2}, \cdots, w_{b-a+2}\right\}$ is a minimum restrained monophonic set and a minimum restrained double monophonic set of $G$ so that $m_{r}(G)=d m_{r}(G)=b$.

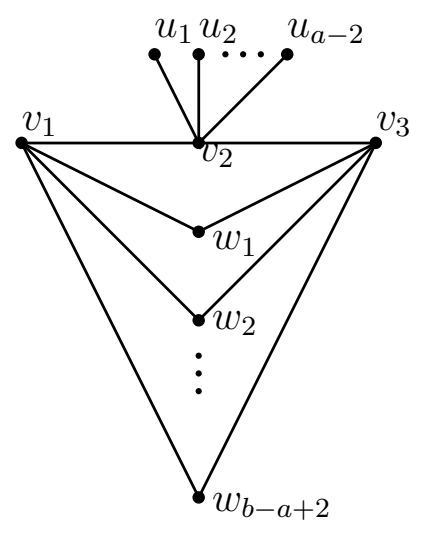

Figure 4. Graph $G$.

Case 4. $a<b<c$. Let $G$ be the graph in Fig. 5 formed from the path $P_{3}: x, y, z$ of order 3 by adding $c$ new vertices $u_{1}, u_{2}, \ldots, u_{a-2}, w_{1}, w_{2}, \ldots, w_{b-a}, v_{1}, v_{2}, \ldots, v_{c-b+2}$ to $P_{3}$ and joining each vertex $v_{i}(1 \leq i \leq c-b+2)$ to the vertices $x, y$ and $z$; joining each vertex $w_{j}(1 \leq j \leq b-a)$ to the 
vertices $x$ and $z$; joining each vertex $u_{i}(1 \leq k \leq a-2)$ to the vertex $y$. By Theorems 1,4 and 6 , every monophonic set, every restrained monophonic set and every restrained double monophonic set of $G$ contain the set $S=\left\{u_{1}, u_{2}, \cdots, u_{a-2}\right\}$ of all extreme vertices of $G$. Clearly, $S$ is not a monophonic set of $G$ and also for any $u \in V(G)-S, S \cup\{u\}$ is not a monophonic set of $G$. It is easily verified that $S_{1}=S \cup\{x, z\}$ is a minimum monophonic set of $G$ and so $m(G)=a$. Since the subgraph induced by $V-S_{1}$ has the isolated vertices $w_{2}, w_{3}, \cdots, w_{b-a}, S_{1}$ is not a restrained monophonic set of $G$. It is clear that every restrained monophonic set of $G$ contains $\left\{w_{2}, w_{3}, \cdots, w_{b-a}\right\}$. Then $S_{2}=S_{1} \cup\left\{w_{1}, w_{2}, \ldots, w_{b-a}\right\}$ is a minimum restrained monophonic set of $G$ and so $m_{r}(G)=b$.

Now, any double monophonic set of $G$ should contain the set $S$. It is easily verified that the set $S^{\prime}=\left\{u_{1}, u_{2}, \cdots, u_{a-2}, w_{1}, w_{2}, \ldots, w_{b-a}, v_{1}, v_{2}, \ldots, v_{c-b+2}\right\}$ is the unique minimum double monophonic set of $G$. Since the subgraph induced by $V-S^{\prime}$ has no isolated vertices, it follows that $S^{\prime}$ is a minimum restrained double monophonic set of $G$ so that $d m_{r}(G)=c$.

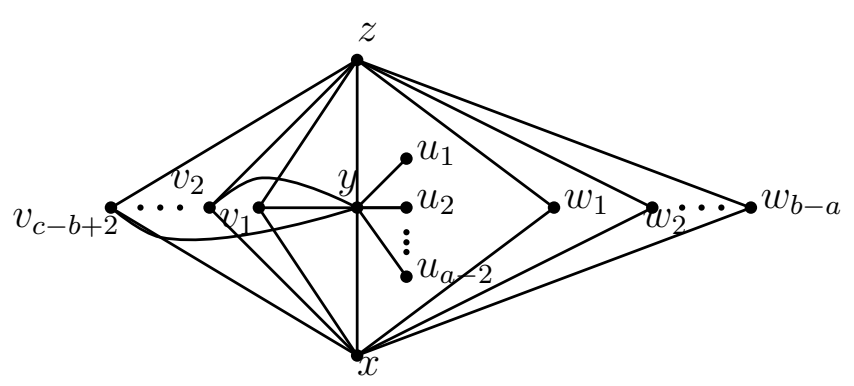

Figure 5. Graph $G$.

Theorem 10. For any integer $p \geq 4$ with $2 \leq k \leq p, k \neq p-1$, there is a connected graph $G$ of order $p$ such that $d m_{r}(G)=k$.

P r o o f. Let $G \neq K_{1, p-1}$ be any tree of order $p$ with $k$ end-vertices. Then clearly, $d m_{r}(G)=k$.

In view of Theorem 8 , we have the following realization theorem.

Theorem 11. For every pair $a, b$ of integers with $3 \leq a \leq b$, there is a connected graph $G$ with $d m_{r}(G)=a$ and $d g_{r}(G)=b$.

P r o o f. For $3 \leq a=b$, by Theorem 5 and Result 1 , the complete graph $K_{a}$ of order $a$ has the desired properties. So, assume that $3 \leq a<b$. Let $H$ be the graph obtained from the complete graph $K_{a-1}$ and the path $P_{4}: u, v, w, x$ of order 4 by joining all the vertices of $K_{a-1}$ to the vertex $u$ of $P_{4}$. Let $G$ be the graph in Fig. 6 obtained from $H$ by taking ' $b-a-1$ ' copies of the path $P_{i}: x_{i}, y_{i}, z_{i}(1 \leq i \leq b-a-1)$ of order 3 and joining each vertex $x_{i}(1 \leq i \leq b-a-1)$ in $P_{i}$ and $u$ in $H$; and also joining each vertex $z_{i}(1 \leq i \leq b-a-1)$ in $P_{i}$ and $w$ in $H$. Let $S=V\left(K_{a-1}\right) \cup\{x\}$ be the set of all extreme vertices of $G$. By Theorems 5 and 6 , every restrained double geodetic set and every restrained double monophonic set of $G$ contain $S$. Clearly, $S$ is the unique minimum restrained double monophonic set of $G$ and so $d m_{r}(G)=a$. Since the pair of vertices $v, y_{i}(i=1,2,3, \cdots, b-a-1)$, do not lie on any geodesic joining a pair of vertices from $S$, $S$ is not a restrained double geodetic set of $G$. Let $S^{\prime}=S \cup\left\{v, y_{1}, y_{2}, \cdots, y_{b-a-1}\right\}$. It is easy to verify that $S^{\prime}$ is a minimum double geodetic set of $G$ and the subgraph induced by $V-S^{\prime}$ has no isolated vertices. Thus $S^{\prime}$ is a minimum restrained double geodetic set of $G$ and so $d g_{r}(G)=b$. 


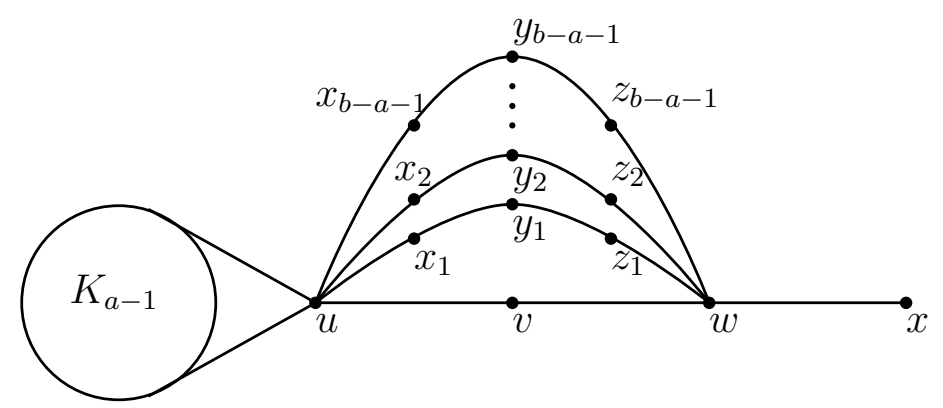

Figure 6. Graph $G$.

Theorem 12. If $G^{\prime}$ is a graph obtained by adding $k$ pendant vertices to a connected graph $G$, then $d m_{r}(G) \leq d m_{r}\left(G^{\prime}\right) \leq d m_{r}(G)+k$.

P r o o f. Let $G^{\prime}$ be the connected graph obtained from $G$ by adding $k$ pendant vertices $v_{i}(1 \leq i \leq k)$ to the vertices $u_{l}(1 \leq l \leq k)$ where each $u_{l}$ is a vertex of $G$ and each $v_{i}(1 \leq i \leq k)$ is not a vertex of $G$. Note that $u_{1}, u_{2}, \cdots, u_{k}$ need not be distinct. Let $S$ be a minimum restrained double monophonic set of $G$. Then $S \cup\left\{v_{1}, v_{2}, \ldots, v_{k}\right\}$ is a restrained double monophonic set of $G^{\prime}$ and so $d m_{r}\left(G^{\prime}\right) \leq d m_{r}(G)+k$.

Now, we claim that $d m_{r}(G) \leq d m_{r}\left(G^{\prime}\right)$. Suppose that $d m_{r}(G)>d m_{r}\left(G^{\prime}\right)$. Let $S^{\prime}$ be a restrained double monophonic set of $G^{\prime}$ with $\left|S^{\prime}\right|<d m_{r}(G)$. Since each $v_{i}(1 \leq i \leq k)$ is an extreme vertex of $G^{\prime}$, it follows from Theorem 6 that $\left\{v_{1}, v_{2}, \ldots, v_{k}\right\} \subseteq S^{\prime}$. Let $S=\left(S^{\prime}-\left\{v_{1}, v_{2}, \ldots, v_{k}\right\}\right) \bigcup\left\{u_{1}, u_{2}, \ldots, u_{l}\right\}(1 \leq l \leq k)$. Then $S$ is a subset of $V(G)$ and $|S| \leq\left|S^{\prime}\right|-k+l=\left|S^{\prime}\right|-(k-l) \leq\left|S^{\prime}\right|<d m_{r}(G)$. Now, we show that $S$ is a restrained double monophonic set of $G$. Let $u, v \in V(G)-S$. Then $u, v \in V\left(G^{\prime}\right)$ also. Since $S^{\prime}$ is a restrained double monophonic set of $G^{\prime}, u$ and $v$ lie on a $x-y$ monophonic path $P$ in $G^{\prime}$ for some vertices $x, y \in S^{\prime}$. If neither $x$ nor $y$ is $v_{i}(1 \leq i \leq k)$, then $x, y \in S$. If exactly one of $x, y$ is $v_{i}(1 \leq i \leq k)$, say $x=v_{i}$, then $u$ and $v$ lie on a $x-y$ monophonic path in $G$, where $y=u_{j}$ and $u_{j}$ is adjacent to $v_{j}$ in $G^{\prime}$ where $i \neq j$. If both $x, y \in\left\{v_{1}, v_{2}, \ldots, v_{k}\right\}$, then let $x=v_{i}$ and $y=v_{j}$ where $i \neq j$. Hence $u$ and $v$ lie on the $u_{s}-u_{t}$ monophonic path in $G$, where $u_{s}$ is adjacent to $v_{i}$ and $u_{t}$ is adjacent to $v_{j}$ in $G^{\prime}$. Thus $S$ is a restrained double monophonic set of $G$. Hence $d m_{r}(G) \leq|S|<d m_{r}(G)$, which is a contradiction.

Remark 3. The bounds for $d m_{r}\left(G^{\prime}\right)$ in Theorem 12 are sharp. Consider a tree $T$ with number of end-vertices $l \geq 3$ and at least two internal vertices. Let $S=\left\{v_{1}, v_{2}, \ldots, v_{l}\right\}$ be the set of all end-vertices of $T$. Then by Result $3, d m_{r}(T)=l$. If we add a pendant vertex to an end-vertex of $T$, then we obtain another tree $T^{\prime}$ with $l$ end-vertices. Hence $d m_{r}(T)=d m_{r}\left(T^{\prime}\right)$. On the other hand, if we add $k$ pendant vertices to a cut-vertex of $T$, then we obtain a tree $T^{\prime}$ with $k+l$ end-vertices. Then by Result $3, d m_{r}\left(T^{\prime}\right)=d m_{r}(T)+k$.

\section{Conclusions}

In this paper, the concept of restrained double monophonic number of a graph is introduced and certain general properties satisfied by this parameter are studied. This parameter is determined for several standard graphs. Also, certain realisation results of this parameter are proved with regard to certain other parameters like monophonic number, restrained monophonic number and restrained double geodetic number of a graph. As a future work of this paper, new parameters like 
upper restrained double monophonic number of a graph, forcing restrained double monophonic number of a graph can be developed and investigated.

\section{Acknowledgement}

The authors are thankful to the reviewers for their useful comments for the improvement of this paper.

\section{REFERENCES}

1. Abdollahzadeh Ahangar H., Samodivkin V., Sheikholeslami S. M. and Abdollah Khodkar. The Restrained Geodetic Number of a Graph. Bull. Malays. Math. Sci. Soc., 2015. Vol. 38. P. 1143-1155. DOI: $10.1007 / \mathrm{s} 40840-014-0068-\mathrm{y}$

2. Buckley F., Harary F. Distance in Graphs. Addison-Wesley, Redwood City, CA, 1990. 335 p.

3. Chartrand G., Harary F. and Zhang P. On the geodetic number of a graph. Networks, 2002. Vol. 39, No. 1. P. 1-6. DOI: $10.1002 /$ net.10007

4. Chartrand G., Johns G. L., and Zhang P. On the detour number and geodetic number of a graph. Ars Combin., 2004. Vol. 72. P. 3-15.

5. Harary F. Graph Theory, Addison-Wesley, 1969.

6. Harary F., Loukakis E. and Tsouros C. The geodetic number of a graph. Math. Comput. Modelling, 1993. Vol. 17, No. 11. P. 89-95. DOI: 10.1016/0895-7177(93)90259-2

7. Santhakumaran A.P. and Jebaraj T. Double geodetic number of a graph. Discuss. Math. Graph Theory, 2012, Vol. 32, No. 1. P. 109-119. DOI: 10.7151/dmgt.1589

8. Santhakumaran A. P., Titus P. and Ganesamoorthy K. On the monophonic number of a graph. J. Appl. Math. Inform., 2014. Vol. 32, No. 1-2. P. 255-266. DOI: 10.14317/jami.2014.255

9. Santhakumaran A.P. and Venakata Raghu T. Double monophonic number of a graph. Int. J. Comput. Appl. Math., 2016. Vol. 11, No. 1. P. 21-26. https://www.ripublication.com/ijcam16/ijcamv11n1_03.pdf

10. Santhakumaran A. P. and Venakata Raghu T. Upper double monophonic number of a graph. Proyecciones, 2018, Vol. 37, No. 2. P. 295-304. DOI: 10.4067/S0716-09172018000200295

11. Santhakumaran A.P. and Venakata Raghu T. Connected double monophonic number of a graph. Int. J. Math. Comb., 2018, Special Issue 1. P. 54-60. 